\title{
The Significance of Propionibacteria in Bone Marrow Cultures
}

\author{
Hisashi Funada, MD, Shinichi Fujita, MD, Kazuo Niwa MD, \\ and Ken-ichi HatTori MD
}

\begin{abstract}
This study was undertaken to reevaluate a hypothesis that human bone marrow may be colonized with propionibacteria. Bacterial cultures were done concurrently when bone marrow aspirations were performed on patients with no evidence of infection. Tincture of iodine, $3 \%$, was applied to the skin at a puncture site. Total contact time with iodine was changed: $2 / 3,3-5,7$, and $13 \mathrm{~min}$, respectively. $16.3 \%$ of a total of 356 sternal marrow cultures from 285 patients were positive. Propionibacteria accounted for $95.0 \%$ of 60 isolates in total. The frequency of positive cultures, regardless of any underlying disease, became significantly reduced, as the disinfection time became longer. Moreover, there were no patients with consecutive positive cultures. On the other hand, a total of 30 iliac marrow cultures from 29 patients proved to be all negative despite relatively short-term disinfection (3-5 min). It was, therefore, concluded that contamination of bone marrow cultures with propionibacteria depends mainly upon their density in sebaceous follicles at puncture sites and that iliac marrow puncture is preferable to sternal one for microbiclogical examinations.
\end{abstract}

Key Words: Propionibacteria, Bone marrow culture, Bone marrow aspiration, Contamination.

Bone marrow cultures are occasionally diagnostic of certain infectious diseases with bacteremia, eg., brucellosis, subacute bacterial endocarditis, histoplasmosis and typhoid fever. However, some workers ${ }^{1,2)}$ stated that propionibacteria were frequently isolated from the sternal marrow of normal subjects or patients without any evidence of infection despite employment of satisfactory skin disinfection procedures. Certainly, we have also had several opportunities of isolating these bacteria from sternal marrow cultures although multiple blood cultures obtained concurrently were all negative. On the other hand, Kassel et al. ${ }^{3)}$ reported that contamination of blood cultures with such organisms could be lowered in frequency to the level of zero with employment of a stringent germ-free technique. This report has prompted us to elucidate why sternal marrow cultures are likely to be contaminated with propionibacteria, which presumably come from the skin flora.

The purpose of this study is to show difficulties in preventing contamination of sternal marrow cultures with propionibacteria despite most adequate skin disinfection, and to preferably recommend iliac marrow punctures for microbiological examinations.

From the Third Department of Internal Medicine, Kanazawa University, School of Medicine, Kanazawa, Ishikawa, Japan.

Received for publication 31 January, 1978.

Present address and Reprint request to: Hisashi Funada, MD. The Third Department of Internal Medicine, Kanazawa University, School of Medicine, 13-1, Takara-machi, Kanazawa, Ishikawa, PC. 920, Japan. 


\section{MATERIALS AND METHODS}

Bone marrow aspiration. Bone marrow aspiration was performed at the outpatient treatment room of our clinic on the patients who had its indication. The upper portion of the sternum, as well as the iliac-crest near the anterior spine, was used for the puncture. The bone marrow was aspirated by the coventional technique ${ }^{4}$. The patients of below 15 years of age or with any sign of an infectious process were excluded from this study.

Skin disinfection. Tincture of iodine, 3\%, was applied briskly and widely to the puncture site for $20 \mathrm{sec}$ and allowed to dry. The second application was done in the same way. Iodine was removed with $7 \%$ sodium thiosulfate (wt/vol) in $70 \%$ ethanol (wt/vol). Total contact time with iodine, which corresponds to twice the period of time between the first and second application, was changed in lengths of $2 / 3,3-5,7,13$, and 23 min, which will be referred to as methods A to E, respectively.

Bone marrow cultures. After aspiration of the first $0.2 \mathrm{ml}$ of marrow for hematological study, the second $1.0 \mathrm{ml}$ was drawn with another sterile syringe, and immediately inoculated into a Culture Bottle No. 2 (thioglycolate medium, Eiken). On subcultivation GAM agar medium (Nissui) was used. Anaerobic conditions were provided by the anaerobic jar method with an atmosphere of $80 \% \mathrm{~N}_{2}, 10 \% \mathrm{CO}_{2}$ and $10 \% \mathrm{H}_{2}$ in the presence of cold catalysts (Anaerobic jar KJ-1, Tomy Seiko). All cultures were incubated at $37^{\circ} \mathrm{C}$. Aerobic isolates were identified by the conventional techniques of Cowan and Steel ${ }^{5)}$, and identification of anaerobic isolates were referred to the Anaerobe Laboratory Manual ${ }^{6}$. Anaerobes were identified to the level of the genus by gas-liquid chromatography (Gas Chromatography Model G6A, Shimazu).

Isolation of propionibacteria from the disinfected skin. The subjects studied were 128 healthy males between the ages of 25-30 years. The presternal area for sternal puncture and the area about the anterior superior iliac spine for iliac-crest puncture were chosen for culture. Skin disinfection by each method mentioned above was performed on each group consisting of 18 subjects at the presternal area and of 10 subjects at the iliac-crest area. Samples including control ones were collected by delineating an area $\left(10 \mathrm{~cm}^{2}\right)$ with a sterile template and scrubbing the skin in that area briskly for one min with cotton-tipped throat swabs moistened with saline containing 1\% Tween 80 . The swab tips were cut off by sterile scissors into $5.0 \mathrm{ml}$ of the same solution in test tubes and agitated on a shaker for $2 \mathrm{~min}$ to dislodge the bacteria. Bacterial counts were made by the conventional pour-plate method, and for estimation of the propionibacteria GAM agar plates were streaked with a loopful of the original suspension.

\section{RESULTS}

Sternal marrow cultures. A total of 356 marrow specimens from 285 patients were cultured during the 3-year period from October, 1974. The number of cultures performed on each patient varied form one to 11 . Fiftyeight cultures (16.3\%) from 57 patients proved to be positive: 45 (18.1\%) of 248 cultures from the patients with only one culture and 13 (12.0\%) of a total of 108 cultures from the remaining 37 patients with repeated cultures (11 with one positive and one with two positives). There were no patients with consective positive cultures among the latter group. Evidence of infection was lacking in the patients with positive cultures.

Iliac marrow cultures. A total of 30 marrow specimens from 29 patients were cultured during the same period of time mentioned above. All cultures proved to be negative. The puncture site was disinfected only by method B.

Organisms isolated. Sixty isolates of 4 bacterial species in total were obtained from the 58 positive cultures. Propionibacterium accounted for $95.0 \%$. Peptococcus, Staphylococcus epidermidis and Pseudomonas aerugi- 
nosa were isolated, one culture each. Each of the latter two were isolated together with Propionibacterium.

Effect of prolonged disinfection. As disinfection time became longer, the frequency of positive cultures became significantly lowered from $30 \%$ in method $\mathrm{A}$ to $5.9 \%$ in both methods $\mathrm{C}$ and $\mathrm{D}$ (Table 1 ). The patients with positive cultures were distributed among a wide variety of diseases such as leukemias, lymphomas, myeloma, aplastic anemia, iron-deficiency anemia, hemolytic anemia, pernicious anemia, thrombocytopenic purpura, gastric cancer, hyperthyroidism, Addison's disease and so on. There was no significant difference in frequency between hematologic and non-hematologic disorders, nor between neoplastic and non-neoplastic diseases. Furthermore, any preponderance of a particular disease with positive cultures was not seen.

Isolation of propionibacteria from the disinfected skin. As shown in Fig. 1, the geometric mean density of propionibacteria isolated

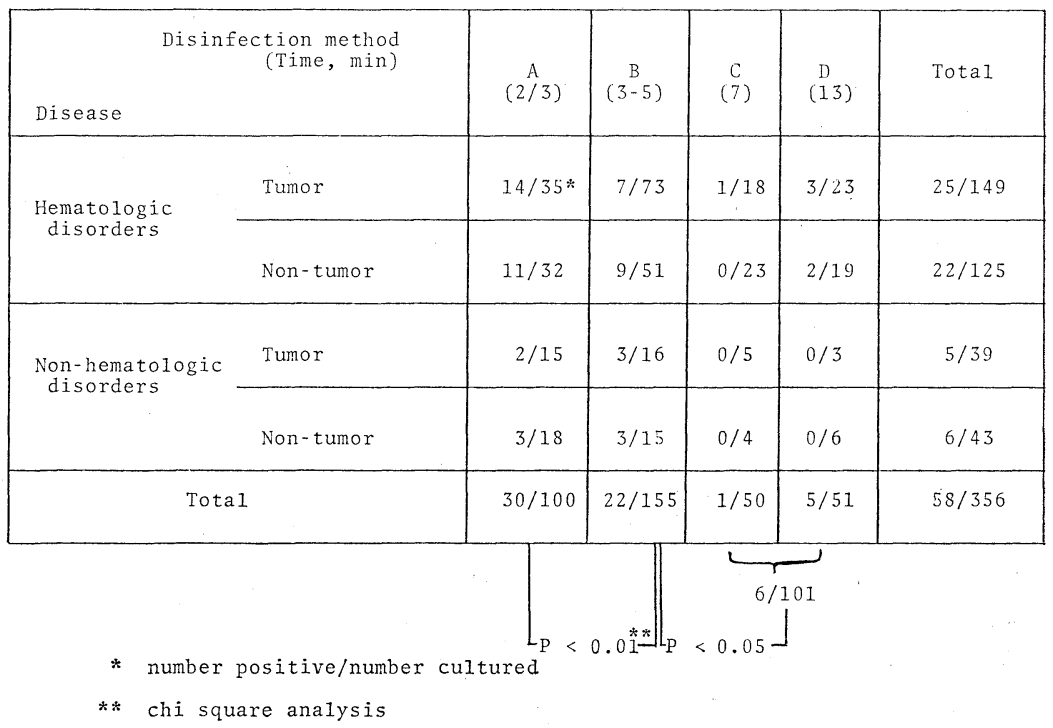

Table 1. Prolonged skin disinfection end positive marrow cultures

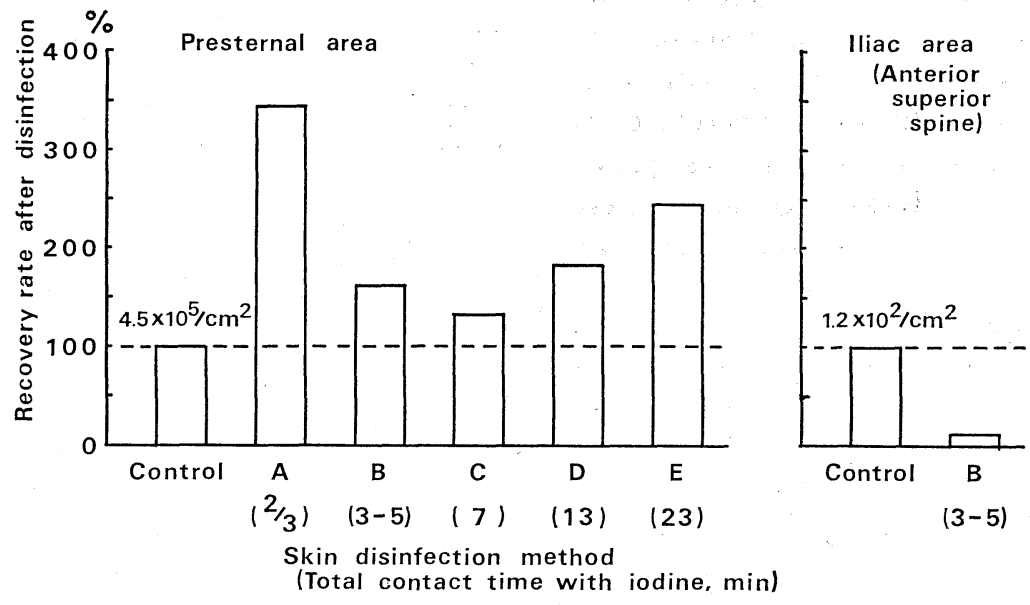

Fig. 1. Recovery of propionibacteria from disinfected skin surface 
from the presternal area was increased about 3.5 times as many after treatment by method $\mathrm{A}$ as the control bacterial count $\left(4.5 \times 10^{5} /\right.$ $\mathrm{cm}^{2}$ ), and it was decreased after treatment by methods $B$ and $C$ in order. But treatment by methods $\mathrm{D}$ and $\mathrm{E}$ inversely resulted in increasing numbers of the organisms isolated. Even with method $\mathrm{C}$ the mean number of propionibacteria isolated was slightly higher than the original value.

In contrast, the distribution of propionibacteria on the iliac area was sparse with a geometric mean density of $1.2 \times 10^{2} / \mathrm{cm}^{2}$. The organisms isolated were reduced about 10 times after treatment by method $\mathrm{B}$.

\section{DISCUSSION}

Some workers ${ }^{1,2)}$ made a practical hypothesis that propionibacteria are situated in the normal bone marrow of man because of their frequent isolation from sternal marrow cultures in spite of rigorous use of a germfree technique. Moreover, Fleisher ${ }^{7}$ suggested that these organisms are present in the blood stream of patients with lymphoreticulo-endothelial disorders. Certainly 58 $(16.3 \%)$ of all the sternal marrow cultures obtained in this study grew 60 bacterial strains, among which Propionibacterium was the most commonly encountered, accounting for $95.0 \%$. But the frequency of positive sternal marrow cultures became significatly reduced as disinfection time with iodine tincture became longer, and it had no relationship with any underlying disease. In addition, there were no patients with consecutive positive cultures. Therefore, it seems reasonable to suppose that, as previously reported by Kassel et al. $^{3)}$, these bacteria came from some source other than the bone marrow itself.

Propionibacteria occur in large numbers in sebaceous follicles which are distributed abundantly in midline areas of the body such as on the face, presternum and upper back, and sparsely on the skin of the legs and $\operatorname{arms}^{8}$. The organisms are found at or near the skin surface. But a significant proportion of them are located so deep in sebaceous follicles that they are beyond the reach of adequate disinfection ${ }^{9)}$. On the other hand, the tincture contains alcohol, which may promote sebum removal on its application. About 50\% of the sebum removal from the skin is replaced within one hour ${ }^{8)}$. We, therefore, inferred that prolongation of total contact time with the tincture stimulated sebum replacement inevitably accompained by discharge of deeperseated propionibacteria, which probably brought about the apparently conflicting results of both increase in recovery of the organisms from disinfected skin and decrease in positive marrow cultures. However, it would be impossible for all practical purposes to entirely prevent contamination of sternal marrow cultures with propionibacteria because more prolonged disinfection with iodine tincture leads to more serious contact dermatitis.

Mori $^{2)}$ reported that $45.4 \%$ of 315 sternal marrow cultures were positive for propionibacteria, while 121 iliac marrow cultures were all negative. Our additional small series of 30 iliac marrow cultures obtained by method $B$ were all negative. These findings are compatible with a sparse population of the organisms at the iliac area near the anterior spine because it seems quite illogical to assume that they are located in the sternal marrow, not in the iliac one. Accordingly, we think that iliaccrest puncture is preferable to sternal one for microbiological examinations.

\section{REFERENCES}

1) Kosakai N, Suzuki S: Anaerobes in clinical medicine. Igaku Shoin, Ltd. Tokyo, 1968.

2) Mori A: Antitumor activity of anaerobic corynebacteria isolated from the human bone marrow. Fukuoka Acta Medica 63: 494-511, 1972.

3) Kassel R, Rottino A : Significance of diphtheroids in malignant disease studied by germfree techniques. Arch Intern Med 96: 804808, 1955.

4) Wintrobe MM : Clinical hematology. 6th ed./ Asian ed., Lea \& Febiger, Philadelphia, 1967/ Igaku Shoin, Tokyo, 1967.

Jap J Med Vol 18, No 1 (Jan 1979) 
5) Cowan ST, Steel KJ: Manual for the identification of medical bacteria. Cambridge University Press, Cambridge, 1970.

6) Holdeman LV, Moore WEG (Eds): Anaerobe Laboratory Manual. Virginia Polytechnic Institute Anaerobe Laboratory, Blacksberg, Va., 1972.

7) Fleisher MS : Significance of diphtheroid microorganisms in blood cultures from human beings. Amer J Med Sci 224 : 548-553, 1952.

8) Noble WC, Somerville DA: Microbiology of human skin. WB Saunders Co. Ltd., London, 1974.

9) Selwyn S, Ellis H: Skin bacteria and skin disinfection reconsidered. Brit Med J 1 : 136140, 1972. 